\title{
Single-Arm Study of Etanercept in Adult Patients with Moderate to Severe Rheumatoid Arthritis Who Failed Adalimumab Treatment
}

\author{
Louis Bessette - Majed Khraishi · Alan J. Kivitz • Arunan Kaliyaperumal • \\ Rama Grantab · Melanie Poulin-Costello • Maya Isaila • \\ David Collier
}

Received: June 21, 2017 / Published online: September 12, 2017

(C) The Author(s) 2017. This article is an open access publication

\begin{abstract}
Introduction: To evaluate the efficacy and safety of etanercept treatment in adult patients with moderate to severe rheumatoid arthritis (RA) who failed to respond (primary failure) or lost a satisfactory response (secondary failure) to adalimumab.
\end{abstract}

Methods: All patients discontinued prior adalimumab treatment and continued methotrexate with etanercept $50 \mathrm{mg}$ once weekly for 24 weeks. The primary study endpoint was American College of Rheumatology 20\% improvement criteria (ACR20) at week 12.

Enhanced content To view enhanced content for this article go to http://www.medengine.com/Redeem/ D3FBF060535AD35C.

L. Bessette $(\square)$

Centre de recherche du CHU de Québec, Quebec

City, QC, Canada

e-mail: louis.bessette@crchudequebec.ulaval.ca

M. Khraishi

Memorial University of Newfoundland, St. John's,

NF, Canada

A. J. Kivitz

Altoona Center for Clinical Research, Duncansville, PA, USA

A. Kaliyaperumal · D. Collier

Amgen Inc., Thousand Oaks, CA, USA

R. Grantab · M. Poulin-Costello · M. Isaila

Amgen Canada Inc., Mississauga, ON, Canada
Results: Eighty-five patients (mean age 56.6 years; female $80.0 \%$ ) were evaluated for safety and 84 for efficacy. Thirty (35.7\%) patients achieved ACR20 at week 12; the lower bound of the 95\% confidence interval (CI; 25.6, 46.9) was greater than the prespecified goal of $24 \%$ based on previous research. Improvements from baseline in clinical outcomes and patient-reported outcomes were observed at each study visit. In planned subgroup analyses, patients with anti-adalimumab antibodies and secondary adalimumab failure had the highest ACR20 response to etanercept at week $12(11 / 17$ patients; $64.7 \%)$. Among the patients with secondary adalimumab failure, those with anti-adalimumab antibodies were fivefold more likely to have an ACR20 response to etanercept than those without anti-adalimumab antibodies (odds ratio 5.2; 95\% CI 2.0, 13.5; $P<0.001$ ). Adverse events were reported for $62(72.9 \%)$ patients and were consistent with previous studies of etanercept. Most adverse events were mild or moderate in severity.

Conclusion: Switching to etanercept is a therapeutic option in patients with RA who fail adalimumab treatment. The presence of anti-adalimumab antibodies may provide additional support for switching to etanercept, particularly in patients with secondary adalimumab failure.

Trial Registration: ClinicalTrials.gov identifier, NCT01927757. 
Keywords: Adalimumab;

Antibodies;

Antirheumatic agents; Etanercept; Humanized; Monoclonal; Receptors; Tumor necrosis factor; Rheumatoid arthritis

\section{INTRODUCTION}

Rheumatoid arthritis (RA) is a chronic, systemic, autoimmune disease that affects approximately $1 \%$ of adults worldwide [1]. Patients with RA experience pain and stiffness in addition to comorbidities that may restrict the patient's physical function and impair their ability to work [2-4]. Treatment guidelines recommend monotherapy with a conventional synthetic disease-modifying antirheumatic drug (csDMARD), preferably methotrexate, as the initial treatment for RA $[5,6]$. Patients with moderate to severe disease activity who do not achieve treatment targets with a csDMARD alone may benefit from the addition of a tumor necrosis factor inhibitor (TNFi) to their therapeutic regimen $[5,6]$.

Biologic agents, such as TNFi, have been a major advancement in the management of RA. However, approximately $25 \%$ of patients discontinue adalimumab or infliximab treatment within 0.8 years of initiation [7]. It has been estimated that $20-30 \%$ of RA patients fail to respond to the first $\mathrm{TNFi}$, and $>20 \%$ of patients who initially respond experience a loss of effectiveness within the first 2 years of treatment [8]. Interestingly, it has long been shown that in patients with secondary TNFi failure, switching to another TNFi may restore clinical response $[5,6,9-14]$, suggesting that the problem is not the drug's mechanism of action and that the loss of efficacy could be related to immunogenicity.

Etanercept and adalimumab are the most commonly used biologics in patients with RA $[15,16]$. An open-label study of patients with RA who switched from another TNFi to adalimumab for a variety of reasons reported that adalimumab was effective and well tolerated among patients with primary or secondary etanercept failure [12]. Conversely, an observational study of etanercept treatment among patients with RA who failed either adalimumab or infliximab treatment showed that $79 \%$ of patients achieved a good or moderate European League Against Rheumatism (EULAR) response to etanercept at 28 weeks, but the efficacy results were not provided separately for patients who previously failed adalimumab treatment [17]. A notable finding of the latter study was a higher response rate in patients who had antibodies to infliximab or adalimumab compared with patients who did not have antibodies to the previous TNFi [17]. Antibodies to infliximab or adalimumab develop frequently and may contribute to treatment failure, but they do not cross-react with etanercept [17-20]. Failure type (primary or secondary) for the previous TNFi may also predict response to a different TNFi $[12,20]$. However, the most recent treatment guidelines for RA from EULAR [6] and the American College of Rheumatology (ACR) [5] do not provide recommendations for the use of immunogenicity testing or failure type to guide treatment decisions.

There has been controversy over whether the next treatment after TNFi failure should be another TNFi or a DMARD with another mechanism of action. The ACR guidelines for the treatment of RA [5] include an algorithm for the treatment of established RA. According to the algorithm, patients who fail a single TNFi should receive either another $\mathrm{TNFi} \pm$ methotrexate or a non-TNFi biologic \pm methotrexate as the next therapy.

The objective of this study was to evaluate the efficacy of etanercept, as measured by ACR 20\% improvement criteria (ACR20) at week 12, in adult patients with moderate to severe RA who failed to respond or lost a satisfactory response to adalimumab when used as their first biologic agent. Secondary objectives included examinations of clinical outcomes and patient-reported outcomes, both for all patients and within subgroups according to anti-adalimumab antibody status and adalimumab failure type.

\section{METHODS}

\section{Study Design}

This single-arm interventional study enrolled patients with moderate to severe RA who either 
did not respond to adalimumab (primary failure) or who lost a satisfactory response to adalimumab (secondary failure) when used as the first biologic agent in combination with methotrexate. The study included a screening period of up to 45 days, a 24 -week treatment period with study visits every 4-6 weeks, and a 30-day safety follow-up visit.

All patients were at least 18 years of age and were diagnosed with RA as determined by meeting the 1987 ACR classification criteria [21] for at least 6 months. At screening, patients were required to have a disease activity score based on the Disease Activity Score 28-joint count C-reactive protein (DAS28-CRP) of at least 3.2, at least three swollen joints, and at least three tender/painful joints. Primary adalimumab failure was an inability to achieve a satisfactory response, defined as achievement of ACR20 or equivalent as judged by the investigator, with a combination treatment of adalimumab and methotrexate received for at least 3 months. Secondary adalimumab failure was the loss of a prior satisfactory response, defined as prior achievement of ACR20 or equivalent as judged by the investigator, to a combination treatment of adalimumab + methotrexate received for at least 6 months.

A two-tiered approach was used to test for anti-adalimumab antibodies, including a screening assay and a specificity assay. Samples were treated with a low $\mathrm{pH}$ buffer to dissociate antibody-drug complexes and then tested in a validated electrochemiluminescence-based bridging immunoassay. Screened samples with a signal-to-noise ratio greater than the assay cutoff point were confirmed in the specificity assay; samples in the specificity assay that showed a signal-to-noise ratio reduction in the presence of excess soluble adalimumab were reported as positive.

Patients currently receiving treatment with adalimumab at screening completed a 2-week washout prior to receiving the first dose of etanercept. During the treatment period, all patients received etanercept in combination with methotrexate. Etanercept dosing was based on the recommended label dosing for patients with RA-50 mg once weekly, administered subcutaneously by the patient or their caregiver. Methotrexate was continued at the same dose, per local labeling. Patients were required to take methotrexate for at least 12 weeks before baseline, with a stable dose of at least $15 \mathrm{mg}$ weekly for at least 8 weeks before baseline (a stable dose of $10 \mathrm{mg}$ to $<15 \mathrm{mg}$ weekly for at least 8 weeks was permitted if required for tolerability). Continued use of a nonsteroidal anti-inflammatory drug or an oral corticosteroid $(\leq 10 \mathrm{mg} /$ day prednisone equivalent) was permitted if the dose was stable for at least 4 weeks before screening.

The study was conducted in accordance with the principles contained in the Declaration of Helsinki. An institutional review board approved the study protocol for each center. Written informed consent was obtained for each patient at enrollment.

\section{Assessments}

The primary study endpoint was ACR20 at week 12. Secondary efficacy endpoints included ACR20 at week 24 and the following endpoints at weeks 12 and 24: ACR50 and ACR70; and DAS28-CRP score (including change from baseline, improvement of at least 1.2 units, and low disease activity of $<3.2$ units). Change from baseline at weeks 12 and 24 was evaluated for the patient-reported outcomes, including Health Assessment Questionnaire Disability Index (HAQ-DI) score; pain score on a visual analog scale (VAS); Medical Outcomes Short Form 36 (SF-36) Physical Function score; and Work Productivity and Activity Impairment (WPAI) questionnaire scores. Exploratory endpoints included ACR20, ACR50, and ACR70 at weeks 4, 8 , and 18; DAS28-CRP score; change from baseline; improvement of at least 1.2 units and low disease activity of $<3.2$ units at weeks 4,8 , and 18; Clinical Disease Activity Index (CDAI); Simplified Disease Activity Index (SDAI); and swollen joint count at all visits. Safety was assessed from adverse events reported during the treatment period or at the safety follow-up visit.

\section{Statistical Methods}

Statistical analyses were conducted with SAS software (SAS Institute, Cary, NC). Patient 
disposition, demographics, baseline characteristics, efficacy, and safety were summarized descriptively for all patients who received at least one dose of etanercept. Efficacy endpoints were summarized descriptively at each time point as well as change from baseline to each post-baseline time point. Additional descriptive summaries of efficacy endpoints were provided for subgroups according to anti-adalimumab antibody status at baseline (present or absent) and adalimumab treatment failure type (primary or secondary). Missing data were imputed using a last observation carried forward approach, carrying post-baseline values forward to time points with missing observations.

The primary objective was assessed by estimating the proportion and 95\% confidence interval (CI) of patients who achieved ACR20 at week 12. An estimate from a single-arm study is best interpreted in the context of a historical placebo control. In a recent randomized, placebo-controlled study of golimumab in patients with RA who had received another TNFi previously, the ACR20 proportion estimate at 14 weeks in the placebo group was $18 \%(95 \%$ CI 12,24$)$ [13]. The upper bound of the $95 \%$ CI (24\%) was assumed to be the historical placebo control proportion. The sample size was justified on observing a level of precision based on a 95\% CI for the primary objective that was strictly greater than $24 \%$.
Approximately 250 patients were planned for this estimation study.

All endpoints were assessed for subgroups of patients with and without anti-adalimumab antibodies at baseline and by adalimumab failure type. ACR20 at weeks 12 and 24 was modeled using logistic regression with covariates for anti-adalimumab antibody status (present or absent), adalimumab failure type (primary or secondary), body mass index at screening, duration of RA at baseline, duration of prior adalimumab treatment, and time since the end of adalimumab treatment. An exploratory analysis using generalized estimating equations for ACR20 response at all measurements (weeks $4,8,12,18$, and 24) was conducted with interactions for anti-adalimumab antibody status and adalimumab failure type.

\section{RESULTS}

\section{Patient Characteristics}

Ninety patients were enrolled at 30 centers in the USA and Canada between May 2013 and December 2014, before the study enrollment was stopped early due to slow enrollment. The last patient received the last on-study dose of etanercept in May 2015. Patient disposition is shown in Fig. 1. Four patients were excluded

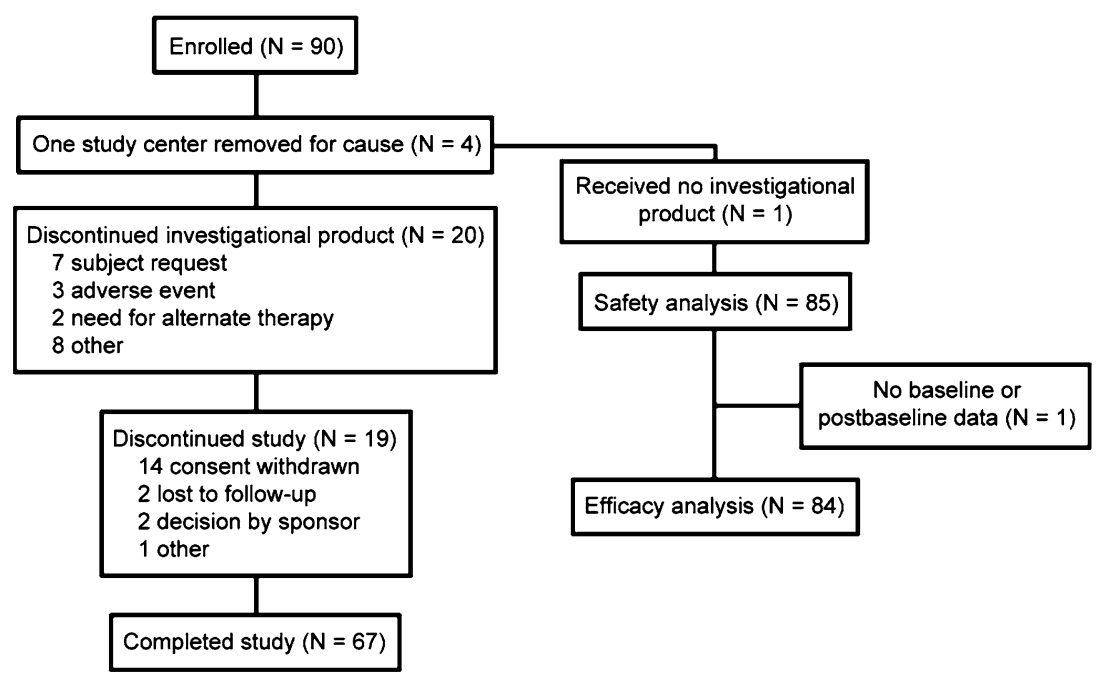

Fig. 1 Flow diagram of patient enrollment and disposition 
from all analyses because of issues with study procedures and the reliability of data at their study center. Of the 86 patients included in the intent to treat (ITT) analysis, 67 (77.9\%) completed the study, and 19 (22.1\%) discontinued the study. The safety analyses included 85 patients (1 ITT patient did not receive any study treatment), and the efficacy analyses included
84 patients (1 patient who received study treatment did not provide efficacy data).

Patient characteristics for the 85 safety-evaluable patients are summarized in Table 1. Anti-adalimumab antibody status at baseline was "positive" (present) in 24 patients (seven with primary adalimumab failure and 17 with secondary adalimumab failure), "negative"

Table 1 Baseline characteristics, overall and by anti-adalimumab antibody status

\begin{tabular}{|c|c|c|c|}
\hline Characteristic & $\begin{array}{l}\text { Anti-ADA antibody } \\
\text { present }(n=24)^{\mathrm{a}}\end{array}$ & $\begin{array}{l}\text { Anti-ADA antibody } \\
\text { absent }(n=56)^{\mathrm{a}}\end{array}$ & Total $(n=85)$ \\
\hline Sex, female & $20(83.3 \%)$ & $44(78.6 \%)$ & $68(80.0 \%)$ \\
\hline Hispanic/Latino & $6(25.0 \%)$ & $7(12.5 \%)$ & $16(18.8 \%)$ \\
\hline \multicolumn{4}{|l|}{ Race } \\
\hline White & $21(87.5 \%)$ & $49(87.5 \%)$ & $74(87.1 \%)$ \\
\hline Black & $2(8.3 \%)$ & $3(5.4 \%)$ & $5(5.9 \%)$ \\
\hline Asian & $1(4.2 \%)$ & $2(3.6 \%)$ & $3(3.5 \%)$ \\
\hline Other & $0(0.0 \%)$ & $2(3.6 \%)$ & $3(3.5 \%)$ \\
\hline Age (years) & $56.5 \pm 9.0$ & $56.5 \pm 12.3$ & $56.6 \pm 11.1$ \\
\hline Patients aged $\geq 65$ years & $6(25.0 \%)$ & $10(17.9 \%)$ & $18(21.2 \%)$ \\
\hline Body mass index $\left(\mathrm{kg} / \mathrm{m}^{2}\right)$ & $32.2 \pm 7.8$ & $34.0 \pm 19.5$ & $33.5 \pm 16.3$ \\
\hline \multicolumn{4}{|l|}{ ADA failure type } \\
\hline Primary & $7(29.2 \%)$ & $22(39.3 \%)$ & $33(38.8 \%)$ \\
\hline Secondary & $17(70.8 \%)$ & $34(60.7 \%)$ & $52(61.2 \%)$ \\
\hline $\begin{array}{l}\text { Duration of rheumatoid arthritis } \\
\text { (years) }\end{array}$ & $4.9[1.2-24.8]$ & $3.6[0.6-18.5]$ & $4.2[0.6-24.8]$ \\
\hline $\begin{array}{l}\text { Duration of prior ADA therapy } \\
\text { (months) }\end{array}$ & $13.0[3.0-107.1]$ & $10.9[3.0-104.6]$ & $10.6[2.8-107.1]$ \\
\hline Time off ADA at baseline (weeks) & $6.0[2.1-25.7]$ & $7.0[2.1-75.3]$ & $6.6[2.1-75.3]$ \\
\hline Baseline DAS28-CRP & $6.0 \pm 1.2$ & $5.6 \pm 1.3$ & $5.7 \pm 1.3$ \\
\hline Baseline CRP (mg/dL) & $1.78 \pm 2.30$ & $0.63 \pm 1.15$ & $0.94 \pm 1.61$ \\
\hline Patient global assessment & $65.7 \pm 21.2$ & $54.4 \pm 21.9$ & $58.2 \pm 21.9$ \\
\hline CDAI & $42.2 \pm 16.5$ & $44.3 \pm 19.7$ & $43.7 \pm 18.7$ \\
\hline
\end{tabular}

Data in table are presented as the mean \pm standard deviation $(\mathrm{SD})$, the median with the range in square brackets, or a number with the percentage in parentheses

$A D A$ Adalimumab, CDAI Clinical Disease Activity Index, DAS28-CRP Disease Activity Score using 28-joint count and C-reactive protein

${ }^{a}$ Of the 85 patients evaluable for safety, anti-ADA antibody status was not tested for 5 patients (4 with primary ADA failure and 1 with secondary ADA failure) 
(absent) in 56 patients ( 22 with primary adalimumab failure and 34 with secondary adalimumab failure), and "not tested" for five patients (four with primary adalimumab failure and one with secondary adalimumab failure). The mean [standard deviation (SD)] age of study participants was 56.6 (11.1) years. Most patients were female $(80.0 \%)$, white $(87.1 \%)$, and not Hispanic $(81.2 \%)$. The median duration of RA at study enrollment was 4.2 (range 0.6-24.8) years. The median duration of prior adalimumab treatment was 10.6 (range 2.8-107.1) months. The median time off adalimumab at baseline was 6.6 (range 2.1-75.3) weeks. The mean (SD) DAS28-CRP score at baseline was 5.7 (1.3). Patients with anti-adalimumab antibodies at baseline were more likely to have secondary adalimumab failure than those without anti-adalimumab antibodies (70.8 vs. $60.7 \%)$, and they had a longer duration of RA (median 4.9 vs. 3.6 years) and a longer history of adalimumab treatment (median 13.0 vs. 10.9 months) (Table 1$)$.

\section{Efficacy}

Among the 84 patients evaluable for efficacy, the primary study endpoint, ACR20 response at week 12, was achieved by 30 (35.7\%) patients, and the lower bound of the 95\% CI $(25.6,46.9)$ was greater than the historical placebo control of $24 \%$. Improvements from baseline in clinical outcomes and patient-reported outcomes were observed at each study visit (Table 2).

In the planned subgroup analyses, improvements from baseline at weeks 12 and 24 of

Table 2 Efficacy of etanercept among patients who previously failed adalimumab treatment $(n=84)$

\begin{tabular}{lllllll}
\hline Outcome $^{\mathbf{a}}$ & Baseline & Week 4 & Week 8 & Week 12 & Week 18 & Week 24 \\
\hline \% with ACR20 response & NA & 19.0 & 29.8 & 35.7 & 31.0 & 34.5 \\
\% with ACR50 response & NA & 4.8 & 9.5 & 10.7 & 14.3 & 15.5 \\
\% with ACR70 response & NA & 0.0 & 1.2 & 2.4 & 7.1 & 3.6 \\
Mean DAS28-CRP & 5.7 & 4.9 & 4.7 & 4.6 & 4.5 & 4.4 \\
Mean CDAI & 43.7 & 33.0 & 30.5 & 28.7 & 28.6 & 26.1 \\
Mean SDAI & 44.5 & 33.9 & 31.2 & 29.6 & 29.3 & 26.9 \\
Mean CRP, mg/dL & 0.94 & 0.78 & 0.71 & 0.84 & 0.59 & 0.70 \\
Mean swollen joint count & 21.8 & 17.0 & 15.8 & 14.3 & 14.9 & 12.7 \\
Mean HAQ-DI & 1.52 & 1.25 & 1.24 & 1.25 & 1.24 & 1.21 \\
Mean HAQ pain VAS & 63.2 & 50.3 & 42.8 & 44.0 & 45.8 & 40.6 \\
Mean SF-36 physical function & 31.4 & NA & NA & 35.9 & NA & 36.5 \\
Mean patient global assessment & 58.2 & 48.0 & 43.4 & 39.8 & 42.4 & 38.3 \\
WPAI (\% work missed) $^{\mathrm{b}}$ & 15.3 & NA & NA & 9.5 & NA & 5.5 \\
WPAI (\% impairment working) & 41.6 & NA & NA & 29.2 & NA & 25.8 \\
WPAI (\% activity impairment) & 57.9 & NA & NA & 43.2 & NA & 40.0 \\
\hline
\end{tabular}

ACR20/50/70 American College of Rheumatology 20\%/50\%/70\% improvement criteria, DI Disability Index, HAQ Health Assessment Questionnaire, NA not assessed, SDAI Simplified Disease Activity Index, SF-36 Medical Outcomes Short Form 36, $V A S$ visual analog scale, WPAI Work Productivity and Activity Impairment

a For the SF-36 physical function domain, increased scores indicate improved health; for other patient-reported outcomes, decreased scores indicate improved health

b Among employed patients $(n=41)$ 
Table 3 Efficacy of etanercept at weeks 12 and 24, by adalimumab failure type

\begin{tabular}{|c|c|c|c|}
\hline Outcome & Time point & Primary ADA failure $(n=33)$ & Secondary ADA failure $(n=52)$ \\
\hline \multicolumn{4}{|l|}{ ACR response ${ }^{a}$} \\
\hline \multirow[t]{2}{*}{ ACR20 response } & Week 12 & $9(27.3 \%)[13.3-45.5]$ & $21(41.2 \%)[27.6-55.8]$ \\
\hline & Week 24 & $7(21.2 \%)[9.0-38.9]$ & $22(43.1 \%)[29.3-57.8]$ \\
\hline \multirow[t]{2}{*}{ ACR50 response } & Week 12 & $1(3.0 \%)[0.1-15.8]$ & $8(15.7 \%)[7.0-28.6]$ \\
\hline & Week 24 & $1(3.0 \%)[0.1-15.8]$ & $12(23.5 \%)[12.8-37.5]$ \\
\hline \multirow[t]{2}{*}{ ACR70 response } & Week 12 & $0(0.0 \%)[-]$ & $2(3.9 \%)[0.5-13.5]$ \\
\hline & Week 24 & $0(0.0 \%)[-]$ & $3(5.9 \%)[1.2-16.2]$ \\
\hline \multicolumn{4}{|c|}{ Mean change from baseline $( \pm S D)^{\mathrm{b}}$} \\
\hline \multirow[t]{2}{*}{ DAS28-CRP } & Week 12 & $-0.8 \pm 1.1$ & $-1.3 \pm 1.2$ \\
\hline & Week 24 & $-1.1 \pm 1.3$ & $-1.5 \pm 1.3$ \\
\hline \multirow[t]{2}{*}{ CDAI } & Week 12 & $-12.3 \pm 14.8$ & $-16.6 \pm 14.2$ \\
\hline & Week 24 & $-14.9 \pm 17.6$ & $-18.8 \pm 13.7$ \\
\hline \multirow[t]{2}{*}{ SDAI } & Week 12 & $-12.5 \pm 15.9$ & $-16.6 \pm 14.3$ \\
\hline & Week 24 & $-15.5 \pm 18.0$ & $-18.8 \pm 13.9$ \\
\hline \multirow[t]{2}{*}{$\mathrm{CRP}(\mathrm{mg} / \mathrm{dL})$} & Week 12 & $-0.18 \pm 2.70$ & $-0.07 \pm 1.42$ \\
\hline & Week 24 & $-0.45 \pm 1.96$ & $-0.12 \pm 0.60$ \\
\hline \multirow[t]{2}{*}{ Swollen joint count } & Week 12 & $-5.9 \pm 13.0$ & $-8.8 \pm 12.0$ \\
\hline & Week 24 & $-9.4 \pm 10.9$ & $-9.2 \pm 14.1$ \\
\hline \multirow[t]{2}{*}{ HAQ-DI } & Week 12 & $-0.10 \pm 0.45$ & $-0.38 \pm 0.57$ \\
\hline & Week 24 & $-0.08 \pm 0.47$ & $-0.46 \pm 0.63$ \\
\hline \multirow[t]{2}{*}{ HAQ VAS } & Week 12 & $-10.2 \pm 23.8$ & $-24.9 \pm 27.8$ \\
\hline & Week 24 & $-15.8 \pm 29.6$ & $-26.7 \pm 25.0$ \\
\hline \multirow[t]{2}{*}{ Patient global assessment } & Week 12 & $-12.4 \pm 24.8$ & $-22.1 \pm 24.2$ \\
\hline & Week 24 & $-13.4 \pm 25.2$ & $-23.9 \pm 26.4$ \\
\hline
\end{tabular}

a ACR response data presented as the number of patients, with the percentage in parentheses and $95 \%$ confidence interval in square brackets

b Decreased scores indicate improved health

etanercept treatment were similar or greater among patients with secondary adalimumab failure $(n=52)$ versus those with primary adalimumab failure $(n=33)$ (Table 3$)$. The ACR20 response rates among patients with primary and secondary adalimumab failure at week 12 were 27.3 and $41.2 \%$, respectively, and at week 24 they were 21.2 and $43.1 \%$, respectively. The ACR50 response rates among patients with primary and secondary adalimumab failure at week 12 were 3.0 and $15.7 \%$, respectively, and at week 24 they were 3.0 and $23.5 \%$, respectively. ACR70 responses were only observed among patients with secondary adalimumab failure.

Patients with both anti-adalimumab antibodies and secondary adalimumab failure at 


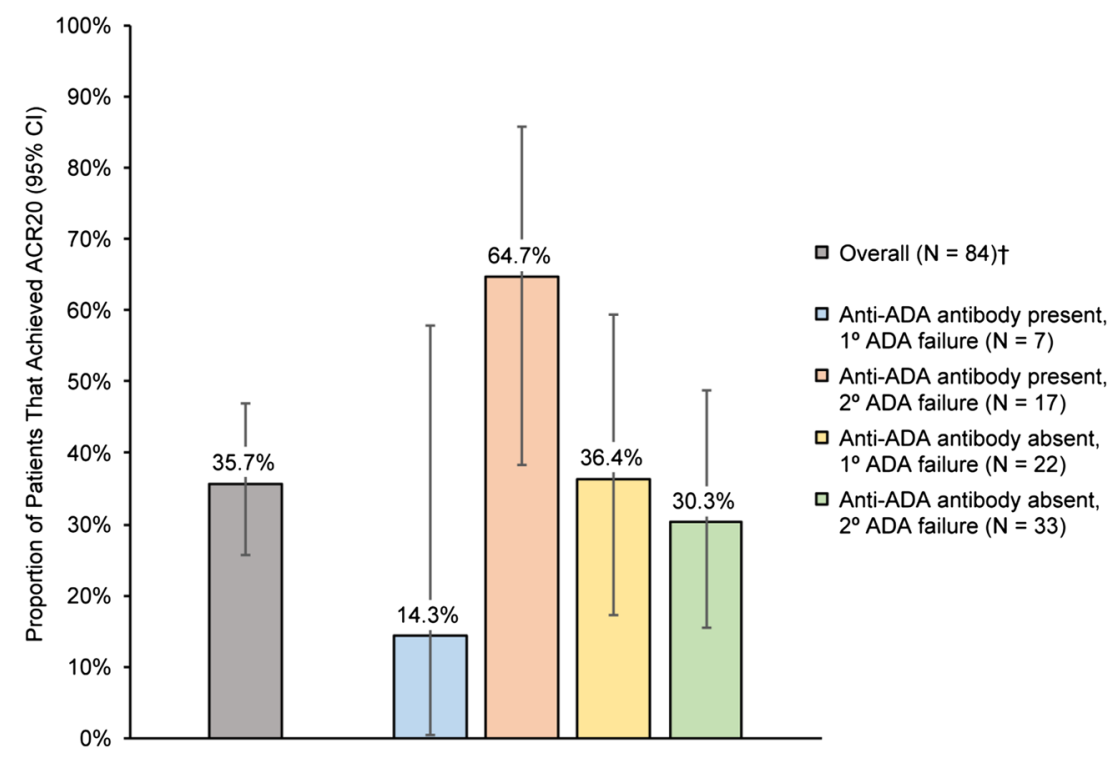

Fig. 2 Etanercept efficacy at week 12, overall and by anti-adalimumab (ADA) antibody status and ADA failure type. Error bars are the $95 \%$ confidence interval. The primary study endpoint, American College of Rheumatology $20 \%$ improvement criteria (ACR20) at week 12, was achieved by $35.7 \%$ of patients; the lower bound of the $95 \%$

baseline had the highest rate of ACR20 response to etanercept at week 12 (11/17 patients; 64.7\%) (Fig. 2). A significant interaction was observed in generalized estimating equation analyses, such that among the patients with secondary adalimumab failure, those with anti-adalimumab antibodies were fivefold more likely to have an ACR20 response to etanercept than those without anti-adalimumab antibodies (odds ratio 5.2; 95\% CI 2.0, 13.5; $P<0.001$ ). At the end of study treatment at 24 weeks, the greatest improvements in clinical outcomes and patient-reported outcomes occurred in patients with anti-adalimumab antibodies and secondary adalimumab failure (Table 4). For example, the HAQ-DI score was reduced (improved) by a mean of 0.31 points from baseline to week 24 .

\section{Safety}

The median duration of on-study etanercept treatment was 162 (range 22-190) days. Adverse events were reported for $62(72.9 \%)$ patients. confidence interval (CI) was $>24 \%$. Missing data were imputed as ACR20 nonresponse. $1^{\circ} / 2^{\circ}$ indicates primary/ secondary ADA failure. Dagger symbol indicates that of the 84 patients evaluable for efficacy, anti-ADA antibody status was not tested for 5 patients (4 with primary ADA failure and 1 with secondary ADA failure)

Adverse events reported for more than $5 \%$ of patients, regardless of severity or relationship to etanercept treatment, were as follows: arthralgia (eight patients, 9.4\%); injection site erythema and nausea (seven patients each, 8.2\%); bronchitis, headache, injection site reaction, and sinusitis (six patients each, 7.1\% each); RA (five patients, $5.9 \%$ ).

Investigators reported that at least one adverse event was related to etanercept treatment for $26(30.6 \%)$ patients. The most commonly reported terms for treatment-related adverse events were injection site erythema (seven patients, 8.2\%), injection site pruritus (four patients, $4.7 \%$ ), and injection site reaction (four patients, 4.7\%). Five events (osteoarthritis, RA, nausea, vomiting, and injection site reaction, respectively) in four $(4.7 \%)$ patients led to discontinuation of etanercept treatment.

Most adverse events were mild or moderate in severity. There were no fatal adverse events and no deaths. Serious adverse events were reported for four $(4.7 \%)$ patients, including one patient with three serious adverse events (abdominal abscess, sepsis, and urinary tract 
Table 4 Efficacy of etanercept at week 24, by anti-adalimumab antibody status and adalimumab failure type

\begin{tabular}{|c|c|c|c|c|}
\hline \multirow[t]{2}{*}{ Outcome } & \multicolumn{2}{|c|}{ Anti-ADA antibody present } & \multicolumn{2}{|c|}{ Anti-ADA antibody absent } \\
\hline & $\begin{array}{l}\text { Primary ADA } \\
\text { failure }(n=7)\end{array}$ & $\begin{array}{l}\text { Secondary ADA } \\
\text { failure }(n=17)\end{array}$ & $\begin{array}{l}\text { Primary ADA } \\
\text { failure }(n=22)\end{array}$ & $\begin{array}{l}\text { Secondary ADA } \\
\text { failure }(n=33)\end{array}$ \\
\hline \multicolumn{5}{|l|}{ ACR response ${ }^{a}$} \\
\hline ACR20 response & $2(28.6 \%)$ & $12(70.6 \%)$ & $4(18.2 \%)$ & $10(30.3 \%)$ \\
\hline ACR50 response & $0(0.0 \%)$ & $7(41.2 \%)$ & $1(4.5 \%)$ & $5(15.2 \%)$ \\
\hline ACR70 response & $0(0.0 \%)$ & $2(11.8 \%)$ & $0(0.0 \%)$ & $1(3.0 \%)$ \\
\hline \multicolumn{5}{|l|}{ DAS28-CRP response ${ }^{a}$} \\
\hline DAS28-CRP $\geq 1.2$ decrease & $3(42.9 \%)$ & $14(82.4 \%)$ & $9(40.9 \%)$ & $14(42.4 \%)$ \\
\hline DAS28-CRP $<3.2$ & $2(28.6 \%)$ & $5(29.4 \%)$ & $4(18.2 \%)$ & $8(24.2 \%)$ \\
\hline \multicolumn{5}{|c|}{ Mean change from baseline to week $24( \pm S D)^{b}$} \\
\hline DAS28-CRP & $-1.26 \pm 2.1$ & $-2.19 \pm 1.1$ & $-1.20 \pm 1.0$ & $-1.10 \pm 1.2$ \\
\hline CDAI & $-11.1 \pm 17.7$ & $-23.3 \pm 11.3$ & $-17.3 \pm 19.1$ & $-17.0 \pm 14.6$ \\
\hline SDAI & $-12.4 \pm 19.4$ & $-23.8 \pm 11.3$ & $-17.8 \pm 19.0$ & $-16.8 \pm 14.7$ \\
\hline $\mathrm{CRP}(\mathrm{mg} / \mathrm{dL})$ & $-0.43 \pm 3.69$ & $-0.44 \pm 0.69$ & $-0.50 \pm 1.40$ & $0.03 \pm 0.51$ \\
\hline Swollen joint count & $-10.1 \pm 8.0$ & $-10.5 \pm 15.9$ & $-9.6 \pm 12.7$ & $-8.4 \pm 13.5$ \\
\hline HAQ-DI & $-0.05 \pm 0.62$ & $-0.65 \pm 0.58$ & $-0.15 \pm 0.45$ & $-0.37 \pm 0.65$ \\
\hline HAQ VAS & $-13.3 \pm 46.8$ & $-45.2 \pm 20.9$ & $-17.0 \pm 24.6$ & $-17.9 \pm 22.0$ \\
\hline SF-36 Physical Function & $6.0 \pm 11.5$ & $8.3 \pm 7.8$ & $3.5 \pm 5.6$ & $4.3 \pm 7.6$ \\
\hline Patient global assessment & $-8.7 \pm 41.7$ & $-44.1 \pm 17.7$ & $-15.6 \pm 20.0$ & $-14.5 \pm 24.3$ \\
\hline
\end{tabular}

${ }^{a}$ ACR response and DAS28-CRP response data presented as the number of patients, with the percentage in parentheses

b For the SF-36 Physical Function domain, increased scores indicate improved health; for other outcomes, decreased scores indicate improved health

infection) that the investigator considered to be related to etanercept. The investigator did not consider the other serious adverse events (infective cholecystitis, pyelonephritis, altered state of consciousness, seizure, and acute myocardial infarction) to be related to etanercept treatment.

\section{DISCUSSION}

Among patients with RA who failed adalimumab treatment, more than one in three patients $(35.7 \%)$ achieved the primary efficacy endpoint of ACR20 response at week 12 after switching to etanercept treatment. Although the full sample size was not achieved before study enrollment was truncated, the lower bound of the $95 \%$ CI for the primary efficacy endpoint was greater than the historical placebo control of $24 \%$ [13]. Thus, the response to etanercept in this single-arm study was greater than that of placebo in a historical control group, which supported the efficacy of etanercept after adalimumab failure.

In this study, improvements in ACR20 were maintained up to the end of etanercept treatment at 24 weeks (6 months), suggesting that the efficacy of etanercept did not decrease over time. This finding was notable, 
because more than $60 \%$ of patients in this study had secondary adalimumab failure at baseline. Biologic agents are large molecules that are potentially immunogenic, as attested by the development of antidrug antibodies. Given the high individual variability in the pharmacokinetics of the TNFi agents and the difficulty in predicting outcomes by clinical judgment alone, the rationale for therapeutic drug level monitoring of biologics and assessment of antidrug antibodies is strengthening [22, 23].

The development of antidrug antibodies to adalimumab or infliximab may be associated with treatment failure, need for dose increases, immune-mediated adverse effects, and cessation of therapy [18]. Treatment failure and the need for dose increases in these patients appears to be related to decreased concentrations of unbound adalimumab [18, 24]. Increased adalimumab dosing frequency (from once every 2 weeks to once weekly) is recommended when a patient with RA fails adalimumab therapy without methotrexate [25], but increasing the dose may improve treatment responses only in a minority of patients $[18,26]$. Neutralizing antibodies that interfere with the drug's binding site for TNF affect the treatment, not the target. Thus, the presence of anti-adalimumab antibodies does not lead to etanercept failure after switching treatments; in fact, patients with anti-adalimumab antibodies may have a greater response to etanercept after switching therapy [17]. We did not measure the levels of adalimumab in the study. Simultaneous measurement of serum TNF-alpha level and antidrug antibodies might have been a better predictor of response.

In this study, etanercept was particularly effective among patients with anti-adalimumab antibodies and secondary adalimumab failure. Comparisons of results across the subgroups were limited by the small sample sizes of the subgroups, which ranged from seven to 34 patients. However, generalized estimating equations identified a statistically significant interaction among the patients with secondary adalimumab failure, with greater than fivefold odds of ACR20 response to etanercept among those with anti-adalimumab antibodies compared with those without anti-adalimumab antibodies. In the subgroup analyses of other clinical outcomes and patient-reported outcomes, including logistic regression, patients with anti-adalimumab antibodies and secondary adalimumab failure generally had numerically greater improvements than the other subgroups.

The ACR20 response rate for etanercept in this study was lowest for the patients with primary adalimumab failure without anti-adalimumab antibodies, which suggests that the inflammatory process in this subgroup of patients was not driven primarily by TNF. In these patients, RA may have been driven by other cytokines, such as interleukin (IL)- 6 or IL-17. Alternatively, this group of patients may have been more difficult to treat with any therapy because of an intrinsic resistance to treatment or longer disease process, making the disease more entrenched and treatment more difficult. A combination of these factors also may explain why intermediate ACR20 response rates were seen among the patients with either primary adalimumab failure with anti-adalimumab antibodies or secondary adalimumab failure without anti-adalimumab antibodies.

While immunogenicity may explain some differences among the TNFi, the lack of standardized assays has led to great variation in the reported prevalence of antidrug antibodies to TNFi [24]. The specificities and sensitivities of available assays differ, making comparisons across studies difficult and leading to confusion for the clinician trying to understand the role and impact of antidrug antibodies. Thus, although the concept of measuring drug or antidrug antibody levels could help guide dosage adjustments or switching, the value of immunopharmacologic guidance in RA is a topic of ongoing debate, and antidrug antibodies are not used in everyday practice to guide the treatment of patients with RA. To date, there are very few evidence-based recommendations to guide selection or switching of biologic therapies based on drug level and immunogenicity testing after failure of TNFi therapy. When more data become available, the benefits of adopting such an approach would 
need to be balanced against the availability of reliable and reproducible tests and monitoring costs.

In the exploratory analyses of results from each study visit, improvements from baseline for clinical outcomes and patient-reported outcomes were observed at each assessment. At the final visit, the observed improvement in mean HAQ-DI score exceeded -0.22 points, which is the minimal clinically important difference for HAQ-DI [27]. The observed decrease in mean scores on the HAQ pain VAS with etanercept treatment after adalimumab failure also exceeded previously reported values for minimally important improvement in pain among patients with RA $[27,28]$. Based on the WPAI, patient-reported rates of absenteeism (missing work) were low before etanercept treatment and even lower during treatment. Patient-reported presenteeism (impairment while working) was more common at baseline and also improved during etanercept treatment.

Safety results during 24 weeks of open-label etanercept treatment were consistent with the known safety profile of etanercept, and there were no fatal adverse events. Most adverse events were mild or moderate in severity, not considered related to etanercept, and nonserious.

A few limitations of the study are noted. The results of this 24-week study should not be extrapolated to treatment beyond 24 weeks or to other switches between biologic therapies. However, results from numerous studies that have examined switches between various biologics among patients with RA also support the benefits of switching to another TNFi when the initial TNFi therapy fails [5, 6, 9-14, 29, 30]. All patients switched to etanercept, and the study did not include a comparator group of patients who continued adalimumab therapy after primary or secondary adalimumab failure. Patients in this study were considered by their physician to be not responding to adalimumab. Although continued adalimumab treatment may have been effective over time, the recommended approach in clinical practice is to stop the ineffective therapy and switch to another therapeutic agent $[5,6]$. This study also did not examine whether etanercept therapy could be discontinued among patients who achieved low disease activity, but previous research suggests that disease activity may increase when etanercept therapy is withdrawn after a treatment response [31].

\section{CONCLUSIONS}

In conclusion, the results of this study suggest that switching to etanercept could be considered as a therapeutic option in patients who fail adalimumab treatment. The presence of anti-adalimumab antibodies may provide additional support for switching to etanercept, particularly in patients with secondary adalimumab failure.

\section{ACKNOWLEDGEMENTS}

This work was supported by Amgen Inc. Article processing charges were funded by Amgen Inc. All authors had full access to all of the data in this study and take complete responsibility for the integrity of the data and accuracy of the data analysis. All named authors meet the International Committee of Medical Journal Editors (ICMJE) criteria for authorship for this manuscript, take responsibility for the integrity of the work as a whole, and have given final approval for the version to be published. Julie Wang of Amgen Inc. and Jonathan Latham of PharmaScribe, LLC (on behalf of Amgen Inc.) assisted with the preparation and submission of the manuscript.

Disclosures. Louis Bessette: Amgen, AbbVie, Pfizer, UCB, Janssen, Bristol-Myers Squibb, and Roche consultancies, speaking fees, and honoraria; Amgen grant/research support. Majed Khraishi: Amgen consultancy and grant/research support. Alan J. Kivitz: Amgen grant/research support. Arunan Kaliyaperumal: Amgen employment and stock. Rama Grantab: Amgen employment and stock. Melanie Poulin-Costello: Amgen employment and stock. Maya Isaila: Amgen employment and stock. David Collier: Amgen employment and stock. 
Compliance with Ethics Guidelines. The study was conducted in accordance with the principles contained in the Declaration of Helsinki. An institutional review board approved the study protocol for each center. Written informed consent was obtained for each patient at enrollment.

Data Availability. The datasets during and/ or analyzed during the current study are available from the corresponding author on reasonable request.

Open Access. This article is distributed under the terms of the Creative Commons Attribution-NonCommercial 4.0 International License (http://creativecommons.org/licenses/ by-nc/4.0/), which permits any noncommercial use, distribution, and reproduction in any medium, provided you give appropriate credit to the original author(s) and the source, provide a link to the Creative Commons license, and indicate if changes were made.

\section{REFERENCES}

1. Helmick CG, Felson DT, Lawrence RC, Gabriel S, Hirsch R, Kwoh CK, Liang MH, Kremers HM, Mayes MD, Merkel PA, Pillemer SR, Reveille JD, Stone JH. Estimates of the prevalence of arthritis and other rheumatic conditions in the United States. Part I. Arthritis Rheum. 2008;58:15-25. doi:10.1002/art. 23177.

2. Salaffi F, Carotti M, Gasparini S, Intorcia M, Grassi $\mathrm{W}$. The health-related quality of life in rheumatoid arthritis, ankylosing spondylitis, and psoriatic arthritis: a comparison with a selected sample of healthy people. Health Qual Life Outcomes. 2009;7:25. doi:10.1186/1477-7525-7-25.

3. Sokka T. Work disability in early rheumatoid arthritis. Clin Exp Rheumatol. 2003;21:S71-4.

4. Zhang W, Bansback N, Boonen A, Young A, Singh A, Anis AH. Validity of the work productivity and activity impairment questionnaire-general health version in patients with rheumatoid arthritis. Arthritis Res Ther. 2010;12:R177. doi:10.1186/ ar3141.

5. Singh JA, Saag KG, Bridges SL Jr, Akl EA, Bannuru RR, Sullivan MC, Vaysbrot E, McNaughton C, Osani
M, Shmerling RH, Curtis JR, Furst DE, Parks D, Kavanaugh A, O'Dell J, King C, Leong A, Matteson EL, Schousboe JT, Drevlow B, Ginsberg S, Grober J, St Clair EW, Tindall E, Miller AS, McAlindon T. 2015 American College of Rheumatology guideline for the treatment of rheumatoid arthritis. Arthritis Rheumatol. 2016;68:1-26. doi:10.1002/art.39480.

6. Smolen JS, Landewe R, Bijlsma J, Burmester G, Chatzidionysiou K, Dougados M, Nam J, Ramiro S, Voshaar M, van Vollenhoven R, Aletaha D, Aringer M, Boers M, Buckley CD, Buttgereit F, Bykerk V, Cardiel M, Combe B, Cutolo M, van Eijk-Hustings Y, Emery P, Finckh A, Gabay C, Gomez-Reino J, Gossec L, Gottenberg JE, Hazes JMW, Huizinga T, Jani M, Karateev D, Kouloumas M, Kvien T, Li Z, Mariette X, McInnes I, Mysler E, Nash P, Pavelka K, Poor G, Richez C, van Riel P, Rubbert-Roth A, Saag K, da Silva J, Stamm T, Takeuchi T, Westhovens R, de Wit $\mathrm{M}$, van der Heijde D. EULAR recommendations for the management of rheumatoid arthritis with synthetic and biological disease-modifying antirheumatic drugs: 2016 update. Ann Rheum Dis. 2017;76:960-77. doi:10.1136/annrheumdis-2016210715.

7. Neovius $\mathrm{M}$, Arkema EV, Olsson H, Eriksson JK, Kristensen LE, Simard JF, Askling J. Drug survival on TNF inhibitors in patients with rheumatoid arthritis comparison of adalimumab, etanercept and infliximab. Ann Rheum Dis. 2015;74:354-60. doi:10. 1136/annrheumdis-2013-204128.

8. Rubbert-Roth A, Finckh A. Treatment options in patients with rheumatoid arthritis failing initial TNF inhibitor therapy: a critical review. Arthritis Res Ther. 2009;11[Suppl 1]:S1. doi:10.1186/ar2666.

9. Malottki K, Barton P, Tsourapas A, Uthman AO, Liu Z, Routh K, Connock M, Jobanputra P, Moore D, Fry-Smith A, Chen YF. Adalimumab, etanercept, infliximab, rituximab and abatacept for the treatment of rheumatoid arthritis after the failure of a tumour necrosis factor inhibitor: a systematic review and economic evaluation. Health Technol Assess. 2011;15:1-278. doi:10.3310/hta15140.

10. Haraoui B, Keystone EC, Thorne JC, Pope JE, Chen I, Asare CG, Leff JA. Clinical outcomes of patients with rheumatoid arthritis after switching from infliximab to etanercept. J Rheumatol. 2004;31:2356-9.

11. Bingham CO 3rd, Ince A, Haraoui B, Keystone EC, Chon Y, Baumgartner S. Effectiveness and safety of etanercept in subjects with RA who have failed infliximab therapy: 16-week, open-label, observational study. Curr Med Res Opin. 2009;25:1131-42. doi:10.1185/03007990902841010.

12. Bombardieri S, Ruiz AA, Fardellone P, Geusens $P$, McKenna F, Unnebrink K, Oezer U, Kary S, Kupper $\mathrm{H}$, Burmester GR. Effectiveness of adalimumab for 
rheumatoid arthritis in patients with a history of TNF-antagonist therapy in clinical practice. Rheumatology (Oxford). 2007;46:1191-9. doi:10. 1093/rheumatology/kem091.

13. Smolen JS, Kay J, Doyle MK, Landewé R, Matteson EL, Wollenhaupt J, Gaylis N, Murphy FT, Neal JS, Zhou Y, Visvanathan S, Hsia EC, Rahman MU. Golimumab in patients with active rheumatoid arthritis after treatment with tumour necrosis factor alpha inhibitors (GO-AFTER study): a multicentre, randomised, double-blind, placebo-controlled, phase III trial. Lancet. 2009;374:210-21. doi:10. 1016/S0140-6736(09)60506-7.

14. Schiff MH, von Kempis J, Goldblum R, Tesser JR, Mueller RB. Rheumatoid arthritis secondary non-responders to TNF can attain an efficacious and safe response by switching to certolizumab pegol: a phase IV, randomised, multicentre, double-blind, 12-week study, followed by a 12-week open-label phase. Ann Rheum Dis. 2014;73:2174-7. doi:10.1136/annrheumdis-2014-205325.

15. Chastek B, White J, Van Voorhis D, Tang D, Stolshek BS. A retrospective cohort study comparing utilization and costs of biologic therapies and JAK inhibitor therapy across four common inflammatory indications in adult US managed care patients. Adv Ther. 2016;33:626-42. doi:10.1007/s12325016-0312-y.

16. Sauer BC, Teng CC, He T, Leng J, Lu CC, Walsh JA, Shah N, Harrison DJ, Tang DH, Cannon GW. Treatment patterns and annual biologic costs in US veterans with rheumatic conditions or psoriasis. J Med Econ. 2016;19:34-43. doi:10.3111/13696998. 2015.1086774.

17. Jamnitski A, Bartelds GM, Nurmohamed MT, van Schouwenburg PA, van Schaardenburg D, Stapel SO, Dijkmans BA, Aarden L, Wolbink GJ. The presence or absence of antibodies to infliximab or adalimumab determines the outcome of switching to etanercept. Ann Rheum Dis. 2011;70:284-8. doi:10.1136/ard.2010.135111.

18. Bartelds GM, Krieckaert CL, Nurmohamed MT, van Schouwenburg PA, Lems WF, Twisk JW, Dijkmans BA, Aarden L, Wolbink GJ. Development of antidrug antibodies against adalimumab and association with disease activity and treatment failure during long-term follow-up. JAMA. 2011;305:1460-8. doi:10.1001/jama.2011.406.

19. van der Laken CJ, Voskuyl AE, Roos JC, Stigter van Walsum M, de Groot ER, Wolbink G, Dijkmans BA, Aarden LA. Imaging and serum analysis of immune complex formation of radiolabelled infliximab and anti-infliximab in responders and non-responders to therapy for rheumatoid arthritis. Ann Rheum Dis. 2007;66:253-6. doi:10.1136/ard.2006.057406.
20. Virkki LM, Valleala H, Takakubo Y, Vuotila J, Relas $\mathrm{H}$, Komulainen R, Koivuniemi R, Yli-Kerttula U, Mali M, Sihvonen S, Krogerus ML, Jukka E, Nyrhinen S, Konttinen YT, Nordstrom DC. Outcomes of switching anti-TNF drugs in rheumatoid arthritisa study based on observational data from the Finnish Register of Biological Treatment (ROB-FIN). Clin Rheumatol. 2011;30:1447-54. doi:10.1007/ s10067-011-1779-1.

21. Arnett FC, Edworthy SM, Bloch DA, McShane DJ, Fries JF, Cooper NS, Healey LA, Kaplan SR, Liang $\mathrm{MH}$, Luthra HS, et al. The American Rheumatism Association 1987 revised criteria for the classification of rheumatoid arthritis. Arthritis Rheum. $1988 ; 31: 315-24$.

22. Laine J, Jokiranta TS, Eklund KK, Väkeväinen M, Puolakka K. Cost-effectiveness of routine measuring of serum drug concentrations and anti-drug antibodies in treatment of rheumatoid arthritis patients with TNF-alpha blockers. Biologics. 2016;10:67-73. doi:10.2147/BTT.S96982.

23. Jani M, Chinoy H, Warren RB, Griffiths CE, Plant D, Fu B, Morgan AW, Wilson AG, Isaacs JD, Hyrich K, Barton A. Clinical utility of random anti-tumor necrosis factor drug-level testing and measurement of antidrug antibodies on the long-term treatment response in rheumatoid arthritis. Arthritis Rheumatol. 2015;67:2011-9. doi:10.1002/art.39169.

24. van Schouwenburg PA, Rispens T, Wolbink GJ. Immunogenicity of anti-TNF biologic therapies for rheumatoid arthritis. Nat Rev Rheumatol. 2013;9:164-72. doi:10.1038/nrrheum.2013.4.

25. AbbVie Inc. Humira ${ }^{\circledR}$ (adalimumab) prescribing information. North Chicago: AbbVie Inc.; 2017.

26. Blom M, Kievit W, Kuper HH, Jansen TL, Visser H, den Broeder AA, Brus HL, van de Laar MA, van Riel PL. Frequency and effectiveness of dose increase of adalimumab, etanercept, and infliximab in daily clinical practice. Arthritis Care Res (Hoboken). 2010;62:1335-41. doi:10.1002/acr.20211.

27. Bruce B, Fries JF. The Stanford Health Assessment Questionnaire: a review of its history, issues, progress, and documentation. J Rheumatol. 2003;30:167-78.

28. Pope JE, Khanna D, Norrie D, Ouimet JM. The minimally important difference for the health assessment questionnaire in rheumatoid arthritis clinical practice is smaller than in randomized controlled trials. J Rheumatol. 2009;36:254-9. doi:10.3899/jrheum.080479.

29. Gomez-Reino JJ, Carmona L, BIOBADASER Group. Switching TNF antagonists in patients with chronic arthritis: an observational study of 488 patients 
over a four-year period. Arthritis Res Ther. 2006;8:R29. doi:10.1186/ar1881.

30. Chatzidionysiou K, Askling J, Eriksson J, Kristensen LE, van Vollenhoven R, ARTIS Group. Effectiveness of TNF inhibitor switch in RA: results from the national Swedish register. Ann Rheum Dis.
2015;74:890-6. doi:10.1136/annrheumdis-2013204714.

31. Gualtierotti R, Casella F. Is it safe to withdraw etanercept in established rheumatoid arthritis after low disease activity achievement? Intern Emerg Med. 2014;9:223-4. doi:10.1007/s11739-013-1024-9. 